\title{
Bianchi Type-I Dark Energy Cosmology with Power-Law Relation in Brans-Dicke Theory of Gravitation
}

\author{
S. D. Katore ${ }^{1}$ and D. V. Kapse $\mathbb{D}^{2}$ \\ ${ }^{1}$ Department of Mathematics, SGBAU, Amravati 444 602, India \\ ${ }^{2}$ Department of Mathematics, PRMIT\&R, Badnera, Amravati 444 701, India \\ Correspondence should be addressed to D. V. Kapse; dipti.kapse@gmail.com
}

Received 3 February 2018; Revised 17 April 2018; Accepted 4 June 2018; Published 9 July 2018

Academic Editor: Edward Sarkisyan-Grinbaum

Copyright (C) 2018 S. D. Katore and D. V. Kapse. This is an open access article distributed under the Creative Commons Attribution License, which permits unrestricted use, distribution, and reproduction in any medium, provided the original work is properly cited. The publication of this article was funded by $\mathrm{SCOAP}^{3}$.

\begin{abstract}
We have studied the interacting and non-interacting dark energy and dark matter in the spatially homogenous and anisotropic Bianchi type-I model in the Brans-Dicke theory of gravitation. The field equations have been solved (i) by using power-law relation and (ii) by assuming scale factor in terms of redshift. Here we have considered two cases of an interacting and non-interacting dark energy scenario and obtained general results. It has been found that for suitable choice of interaction between dark energy and dark matter we can avoid the coincidence problem which appears in the $\Lambda C D M$ model. Some physical aspects and stability of the models are discussed in detail. The statefinder diagnostic pair, i.e., $\{r, s\}$, is adopted to differentiate our dark energy models.
\end{abstract}

\section{Introduction}

The recent cosmological observational data of Type Ia Supernovae (SNeIa) (Riess et al. [1]; Perlmutter et al. [2]), Cosmic Microwave Background (CMB) (Bennett et al. [3]; Spergel et al. [4]), Large Scale Structure (LSS) (Tegmark et al. [5, 6]), the Sloan Digital Sky Survey (SDSS) (Seljak et al. [7], AdelemanMcCarthy et al. [8]), Wilkinson Microwave Anisotropy Probe (WMAP) (Knop et al. [9]), and Chandra X-ray observatory (Allen et al. [10]) strongly suggests that our universe is dominated by a component with large negative pressure called dark energy (DE).

The study of $\mathrm{DE}$ is possible through its equation of state (EoS) parameter $\omega^{d e}=p^{d e} / \rho^{d e}$ which is not necessarily constant, where $p^{d e}$ is the pressure and $\rho^{d e}$ is the energy density of DE. The DE candidate which can simply explain the cosmic acceleration is a vacuum energy $\left(\omega^{d e}=-1\right)$, which is mathematically equivalent to the cosmological constant $(\Lambda)$. The other conventional alternatives, which can be described by minimally coupled scalar fields, are quintessence $(-1<$ $\left.\omega^{d e}<-1 / 3\right)$, phantom $\left(\omega^{d e}<-1\right)$, and quintom (that can cross from phantom region to quintessence region). From observational results coming from $\mathrm{SNe}$ Ia data (Knop et al.
[9]) and combination of SNe Ia data with CMBR anisotropy and galaxy clustering statistics (Tegmark et al. [8] ), the limits on EoS parameter are obtained as $-1.67<\omega^{d e}<-0.62$ and $-1.33<\omega^{\text {de }}<-0.79$, respectively. Recently, DE models with variable EoS parameter have been studied by Ram et al. $[11,12]$, Katore et al. [13], Reddy et al. [14], and Mahanta et al. [15].

Interaction between $\mathrm{DE}$ and dark matter (DM) leads to a solution to the coincidence problem (Cimento et al. [16]; Dalal et al. [17]; Jamil and Rashid [18, 19]). By considering a coupling between $\mathrm{DE}$ and $\mathrm{DM}$, we can explain why the energy densities of DE and DM are nearly equal today. Due to interaction between two components, the energy conservation cannot hold for the individual components. Recent observations (Bertolami et al. [20]; Le Delliou et al. [21]; Berger and Shojaei [22]) provide the evidence for the possibility of such an interaction between DE and DM. Zhang [23, 24], Zimdahl and Pavon [25], Pradhan et al. [26, 27], Saha et al. [28], Amirhashchi et al. [29-33], Adhav et al. [34, 35], and Fayaz [36] have investigated various cosmological models with interacting DE.

The Brans-Dicke theory [37] is a generalized form of general relativity and it is one of the most enchanting 
examples of scalar tensor theories of gravitation. BransDicke (BD) theory introduces a scalar field $\phi$ which has the dimensions of the inverse of gravitational constant and which interacts equally with all forms of matter. Recently, Rao et al. [38], Sarkar [39, 40], Katore et al. [41], Singh and Dewri [42], and Reddy et al. [43, 44] have studied the cosmological models in Brans-Dicke theory of gravitation.

Amirhashchi et al. [33] have investigated the DE equation of state (EoS) parameter in both interacting and noninteracting cases and examined its future by applying hyperbolic scale factor in general relativity. Motivated by the above investigations, in this paper, we have extended the work of Amirhashchi et al. [33] in Brans-Dicke theory of gravitation. This is relevant because of the fact that scalar field plays an important role in the discussion of DE models. In this paper, we have studied the interacting and non-interacting dark energy and dark matter in the spatially homogenous and anisotropic Bianchi type-I model in the Brans-Dicke theory of gravitation. The field equations have been solved (i) by using power-law relation and (ii) by assuming scale factor in terms of redshift and have discussed the physical properties and also the physical acceptability and stability of our models. The values of cosmological parameters are taken from the recent observations made by Amirhashchi [45], Amirhashchi and Amirhashchi [46, 47], and Patrignani et al. [48]. The paper has following structure. In Section 2, the metric and the Brans-Dicke field equations are described. Section 3 is devoted to the solution of the field equations. Using the scale factor as a function of redshift, we have obtained our results for non-interacting and interacting cases. In Section 4, we have discussed the physical aspects and stability of models. In Section 5, behavior of anisotropy parameter of expansion $(\Delta)$ is studied. The statefinder diagnostic pair, i.e., $\{r, s\}$, is adopted to characterize different phases of the universe in Section 6 and finally, Section 7 contains some concluding remarks.

\section{The Metric and BD Field Equations}

We consider the homogeneous and anisotropic Bianchi typeI universe as

$$
d s^{2}=-d t^{2}+A^{2} d x^{2}+B^{2} d y^{2}+C^{2} d z^{2},
$$

where the scale factors $A, B$, and $C$ are functions of time $t$ only.

$\mathrm{BD}$ field equations for the combined scalar and tensor fields with $(8 \pi G=c=1)$ are given by (Brans-Dicke [37], Reddy et al. [43], and Rao et al. [49])

$$
\begin{aligned}
R_{i j}- & \frac{1}{2} g_{i j} R-\bar{\omega} \phi^{-2}\left(\phi_{, i} \phi_{, j}-\frac{1}{2} g_{i j} \phi^{, k} \phi_{, k}\right) \\
- & \phi^{-1}\left(\phi_{i ; j}-g_{i j} \phi_{; k}^{, k}\right)=\phi^{-1}\left(T_{i j}\right),
\end{aligned}
$$

where $R$ is the Ricci scalar, $R_{i j}$ is the Ricci tensor, $\phi$ is the Brans-Dicke scalar field, $\bar{\omega}$ is the dimensionless constant, and $T_{i j}$ is the energy momentum tensor. The scalar fields satisfy the following equation:

$$
\phi_{; k}^{, k}=\frac{T_{j}^{i}}{3+2 \bar{\omega}} .
$$

The energy momentum tensor is given by

$$
T_{j}^{i}=T_{j}^{(m) i}+T_{j}^{(d e) i}
$$

where $T_{j}^{(m) i}$ and $T_{j}^{(d e) i}$ are energy momentum tensors of DM and DE, respectively. These are given by

$$
\begin{aligned}
T_{j}^{(m) i} & =\operatorname{diag}\left[-\rho^{m}, p^{m}, p^{m}, p^{m}\right], \\
& =\operatorname{diag}\left[-1, \omega^{m}, \omega^{m}, \omega^{m}\right] \rho^{m}
\end{aligned}
$$

and

$$
\begin{aligned}
T_{j}^{(d e) i} & =\operatorname{diag}\left[-\rho^{d e}, p^{d e}, p^{d e}, p^{d e}\right] \\
& =\operatorname{diag}\left[-1, \omega^{d e}, \omega^{d e}, \omega^{d e}\right] \rho^{d e}
\end{aligned}
$$

where $\rho^{m}$ and $\rho^{d e}$ are energy densities of DM and DE, respectively. Similarly, $p^{m}$ and $p^{d e}$ are the pressure of DM and DE, respectively, while $\omega^{m}=p^{m} / \rho^{m}$ and $\omega^{d e}=p^{d e} / \rho^{d e}$ are the corresponding EoS parameters of DM and DE (Harko et al. [50]).

In comoving coordinate system, the $\mathrm{BD}$ field equations (2) and (3) for the metric (1) are given by

$$
\begin{aligned}
\frac{\dot{A} \dot{B}}{A B} & +\frac{\dot{B} \dot{C}}{B C}+\frac{\dot{A} \dot{C}}{A C}-\frac{\bar{\omega}}{2} \frac{\dot{\phi}^{2}}{\phi^{2}}+\left(\frac{\dot{A}}{A}+\frac{\dot{B}}{B}+\frac{\dot{C}}{C}\right) \frac{\dot{\phi}}{\phi} \\
& =\frac{\rho^{m}+\rho^{d e}}{\phi}, \\
\frac{\ddot{B}}{B}+ & \frac{\ddot{C}}{C}+\frac{\dot{B} \dot{C}}{B C}+\frac{\bar{\omega}}{2} \frac{\dot{\phi}^{2}}{\phi^{2}}+\frac{\ddot{\phi}}{\phi}+\left(\frac{\dot{B}}{B}+\frac{\dot{C}}{C}\right) \frac{\dot{\phi}}{\phi} \\
& =\frac{1}{\phi}\left[-\omega^{m} \rho^{m}-\omega^{d e} \rho^{d e}\right], \\
\frac{\ddot{A}}{A}+ & \frac{\ddot{C}}{C}+\frac{\dot{A} \dot{C}}{A C}+\frac{\bar{\omega}}{2} \frac{\dot{\phi}^{2}}{\phi^{2}}+\frac{\ddot{\phi}}{\phi}+\left(\frac{\dot{A}}{A}+\frac{\dot{C}}{C}\right) \frac{\dot{\phi}}{\phi} \\
& =\frac{1}{\phi}\left[-\omega^{m} \rho^{m}-\omega^{d e} \rho^{d e}\right], \\
\frac{\ddot{A}}{A}+ & \frac{\ddot{B}}{B}+\frac{\dot{A} \dot{B}}{A B}+\frac{\bar{\omega}}{2} \frac{\dot{\phi}^{2}}{\phi^{2}}+\frac{\ddot{\phi}}{\phi}+\left(\frac{\dot{A}}{A}+\frac{\dot{B}}{B}\right) \frac{\dot{\phi}}{\phi} \\
& =\frac{1}{\phi}\left[-\omega^{m} \rho^{m}-\omega^{d e} \rho^{d e}\right],
\end{aligned}
$$

and the wave equation is

$$
\begin{aligned}
\ddot{\phi} & +\left(\frac{\dot{A}}{A}+\frac{\dot{B}}{B}+\frac{\dot{C}}{C}\right) \dot{\phi} \\
& =\frac{\rho^{m}\left(1-3 \omega^{m}\right)+\rho^{d e}\left(1-3 \omega^{d e}\right)}{3+2 \bar{\omega}},
\end{aligned}
$$

where an overhead dot denotes differentiation with respect to $t$ 


\section{Solutions of Field Equations}

We have initially six variables and four linearly independent equations (9)-(12). The system is thus initially undetermined and we need additional condition to solve the system completely. In order to solve these field equations, we first assume the power-law relation between the average scale factor $(a)$ and scalar field $(\phi)$ (Pimental [51], Johri and Desikan [52]) as

$$
\phi=\alpha a^{\beta}
$$

where $\alpha$ and $\beta>0$ are constants.

To examine the general results, we assume that the average scale factor is a hyperbolic function of time as Amirhashchi [53]

$$
a(t)=\sinh (t),
$$

which gives dynamical deceleration parameter (q). Also, Chen and Kao [54] have shown that this scale factor is stable under metric perturbation.

In terms of redshift the above scale factor is given by

$$
a(t)=\frac{1}{1+z}, \quad z=\frac{1}{\sinh (t)}-1,
$$

where $z$ is the redshift parameter and $a=(A B C)^{1 / 3}$ is the average scale factor.

From (10)-(12), we obtain

$$
\begin{aligned}
& \frac{\ddot{A}}{A}-\frac{\ddot{B}}{B}+\frac{\dot{C}}{C}\left(\frac{\dot{A}}{A}-\frac{\dot{B}}{B}\right)+\left(\frac{\dot{A}}{A}-\frac{\dot{B}}{B}\right) \frac{\dot{\phi}}{\phi}=0, \\
& \frac{\ddot{B}}{B}-\frac{\ddot{C}}{C}+\frac{\dot{A}}{A}\left(\frac{\dot{B}}{B}-\frac{\dot{C}}{C}\right)+\left(\frac{\dot{B}}{B}-\frac{\dot{C}}{C}\right) \frac{\dot{\phi}}{\phi}=0, \\
& \frac{\ddot{A}}{A}-\frac{\ddot{C}}{C}+\frac{\dot{B}}{B}\left(\frac{\dot{A}}{A}-\frac{\dot{C}}{C}\right)+\left(\frac{\dot{A}}{A}-\frac{\dot{C}}{C}\right) \frac{\dot{\phi}}{\phi}=0,
\end{aligned}
$$

Solving (17)-(19), we obtain

$$
\begin{aligned}
& \frac{\dot{A}}{A}-\frac{\dot{B}}{B}=\frac{k_{1}}{A B C \phi}, \\
& \frac{\dot{B}}{B}-\frac{\dot{C}}{C}=\frac{k_{2}}{A B C \phi}, \\
& \frac{\dot{A}}{A}-\frac{\dot{C}}{C}=\frac{k_{3}}{A B C \phi},
\end{aligned}
$$

where $k_{1}, k_{2}$, and $k_{3}$ are constants of integration.

Equations (20)-(22) further reduce to

$$
\begin{aligned}
& \frac{A}{B}=d_{1} \exp \left(k_{1} \int \frac{d t}{A B C \phi}\right), \\
& \frac{B}{C}=d_{2} \exp \left(k_{2} \int \frac{d t}{A B C \phi}\right), \\
& \frac{A}{C}=d_{3} \exp \left(k_{3} \int \frac{d t}{A B C \phi}\right),
\end{aligned}
$$

where $d_{1}, d_{2}$, and $d_{3}$ are constants of integration.
Using (23)-(25), we can write the metric functions $A, B$, and $C$ explicitly as

$$
\begin{aligned}
& A=a_{1} a \exp \left(b_{1} \int \frac{d t}{a^{3} \phi}\right), \\
& B=a_{2} a \exp \left(b_{2} \int \frac{d t}{a^{3} \phi}\right), \\
& C=a_{3} a \exp \left(b_{3} \int \frac{d t}{a^{3} \phi}\right),
\end{aligned}
$$

where $a_{1}=\left(d_{1} d_{2}\right)^{1 / 3}, a_{2}=\left(d_{1}^{-1} d_{3}\right)^{1 / 3}, a_{3}=\left(d_{2} d_{3}\right)^{-1 / 3}, b_{1}=$ $\left(k_{1}+k_{2}\right) / 3, b_{2}=\left(k_{3}-k_{1}\right) / 3$, and $b_{3}=-\left(k_{2}+k_{3}\right) / 3$, which satisfies the relations $a_{1} a_{2} a_{3}=1$ and $b_{1}+b_{2}+b_{3}=0$.

Using (26)-(28) in (9), we obtain

$$
H^{2}=\frac{\rho^{m}+\rho^{d e}}{D \phi}+\frac{K}{D} a^{-6} \phi^{-2},
$$

where $K=-\left(b_{1} b_{2}+b_{2} b_{3}+b_{1} b_{3}\right), D=\left(3+\beta-\beta^{2}(\bar{\omega} / 2)\right)$, and $H=(1 / 3)(\dot{A} / A+\dot{B} / B+\dot{C} / C)$ are the Hubble parameters. For $b_{1}=b_{2}=b_{3}=0$, the model reduces to the flat FRW model in $\mathrm{BD}$ theory.

The energy conservation equation $T_{; j}{ }^{i j}=0$ is $T^{(m)}{ }_{; j}{ }^{i j}+$ $T^{(d e)}{ }_{; j}^{i j}=0$ and is given by

$$
\dot{\rho}^{m}+3 \frac{\dot{a}}{a}\left(1+\omega^{m}\right) \rho^{m}+\dot{\rho}^{d e}+3 \frac{\dot{a}}{a}\left(1+\omega^{d e}\right) \rho^{d e}=0 .
$$

3.1. Non-Interacting Dark Energy and Dark Matter. In this section we have considered that there is no interaction between DE and DM. Therefore, the general form of energy conservation equation (30) leads to (Harko et al. [50])

$$
\dot{\rho}^{m}+3 \frac{\dot{a}}{a}\left(1+\omega^{m}\right) \rho^{m}=0
$$

and

$$
\dot{\rho}^{d e}+3 \frac{\dot{a}}{a}\left(1+\omega^{d e}\right) \rho^{d e}=0 .
$$

Using (31), we obtain the energy density of DM as

$$
\begin{aligned}
\rho^{m} & =\rho_{0}^{m} a^{-3\left(1+\omega^{m}\right)} \\
& =\rho_{0}^{m}(1+z)^{3\left(1+\omega^{m}\right)},
\end{aligned}
$$

where $\rho_{0}^{m}>0$ is a constant of integration.

Using (33) and (29), we obtain the energy density of DE as

$$
\begin{aligned}
\rho^{d e} & =D H^{2} \alpha a^{\beta}-\rho_{0}^{m} a^{-3\left(1+\omega^{m}\right)}-K \alpha^{-1} a^{-(\beta+6)} \\
= & \left(1+(1+z)^{2}\right)\left(D \alpha(1+z)^{-\beta}-3 \Omega_{0}^{m}(1+z)^{3\left(1+\omega^{m}\right)}\right) \\
& -K \alpha^{-1}(1+z)^{(\beta+6)},
\end{aligned}
$$

where $\Omega^{m}=\rho^{m} / 3 H^{2}$ is the energy density of DM and 0 denotes the present value of $\Omega^{m}$.

Using (14), (33), and (35) in (10), we obtain the EoS parameter of DE as 


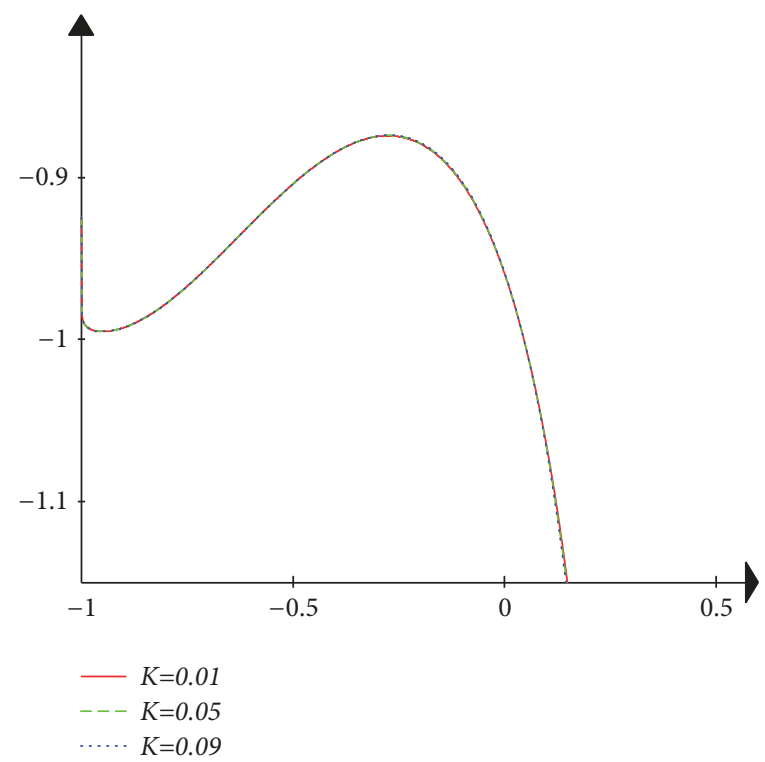

Figure 1: The plot of EoS parameter $\omega^{d e}$ versus redshift $z$ for $\alpha=1, \beta=0.01, \Omega_{0}^{m}=0.3, \omega^{m}=0$, $\bar{\omega}=2$, and vary $K=0.01,0.05,0.09$, resp.

$$
\omega^{d e}=\frac{H^{2}\left[(\beta+2) q-\left(\beta^{2}(1+\bar{\omega} / 2)+\beta+1\right)\right] \alpha a^{\beta}+K \alpha^{-1} a^{-(\beta+6)}-3 \omega^{m} \Omega_{0}^{m} H^{2} a^{-3\left(1+\omega^{m}\right)}}{H^{2}\left(D \alpha a^{\beta}-3 \Omega_{0}^{m} a^{-3\left(1+\omega^{m}\right)}\right)-K \alpha^{-1} a^{-(\beta+6)}} .
$$

where $q=-\ddot{a} / a H^{2}=-1 /\left(1+(1+z)^{2}\right)$ is the deceleration parameter.
Using (16) in (37), we obtain the EoS parameter of DE in terms of redshift as

$$
=-\frac{\left[(\beta+2)\left(1 /\left(1+(1+z)^{2}\right)\right)+\left(\beta^{2}(1+\bar{\omega} / 2)+\beta+1\right)\right] \alpha(1+z)^{-\beta}-K \alpha^{-1}\left((1+z)^{(\beta+6)} /\left(1+(1+z)^{2}\right)\right)+3 \omega^{m} \Omega_{0}^{m}(1+z)^{3\left(1+\omega^{m}\right)}}{D \alpha(1+z)^{-\beta}-K \alpha^{-1}\left((1+z)^{(\beta+6)} /\left(1+(1+z)^{2}\right)\right)-3 \Omega_{0}^{m}(1+z)^{3\left(1+\omega^{m}\right)}} .
$$

The behavior of EoS parameter of DE in terms of redshift $z$ is depicted in Figure 1. Here the parameter $\omega^{m}$ is taken to be zero and vary constant $K$ as $0.01,0.05$, and 0.09 , respectively. From figure it is clear that, for all small values of $K$, the EoS of $\mathrm{DE}$ is varying in quintessence region and crossing Phantom Divide line $(\mathrm{PDL}) \omega^{d e}=-1$. However, it is observed that at late time (i.e., at $z=-1$ ) the EoS parameter $\omega^{d e} \approx-1$. Therefore we say that the cosmological constant is a suitable candidate to represent the behavior of $\mathrm{DE}$ in the derived model at late times.

The matter energy density parameter $\Omega^{m}$ and dark energy density parameter $\Omega^{\text {de }}$ are given by

$$
\begin{aligned}
\Omega^{m} & =\frac{\rho^{m}}{3 H^{2}} \\
& =\Omega_{0}^{m}(1+z)^{3\left(1+\omega^{m}\right)},
\end{aligned}
$$

$$
\begin{aligned}
\Omega^{d e} & =\frac{\rho^{d e}}{3 H^{2}} \\
& =\frac{D \alpha}{3}(1+z)^{-\beta}-\frac{K \alpha^{-1}(1+z)^{(\beta+6)}}{3\left(1+(1+z)^{2}\right)} \\
& -\Omega_{0}^{m}(1+z)^{3\left(1+\omega^{m}\right)} .
\end{aligned}
$$

Using (40) and (42), we obtain overall density parameter $\Omega$ as

$$
\begin{aligned}
\Omega & =\Omega^{m}+\Omega^{d e} \\
& =\frac{D \alpha}{3}(1+z)^{-\beta}-\frac{K \alpha^{-1}(1+z)^{(\beta+6)}}{3\left(1+(1+z)^{2}\right)} .
\end{aligned}
$$

The variation of density parameters $\Omega^{m}$ and $\Omega^{d e}$ with redshift $z$ is depicted in Figure 2. Dot denotes the current value of 


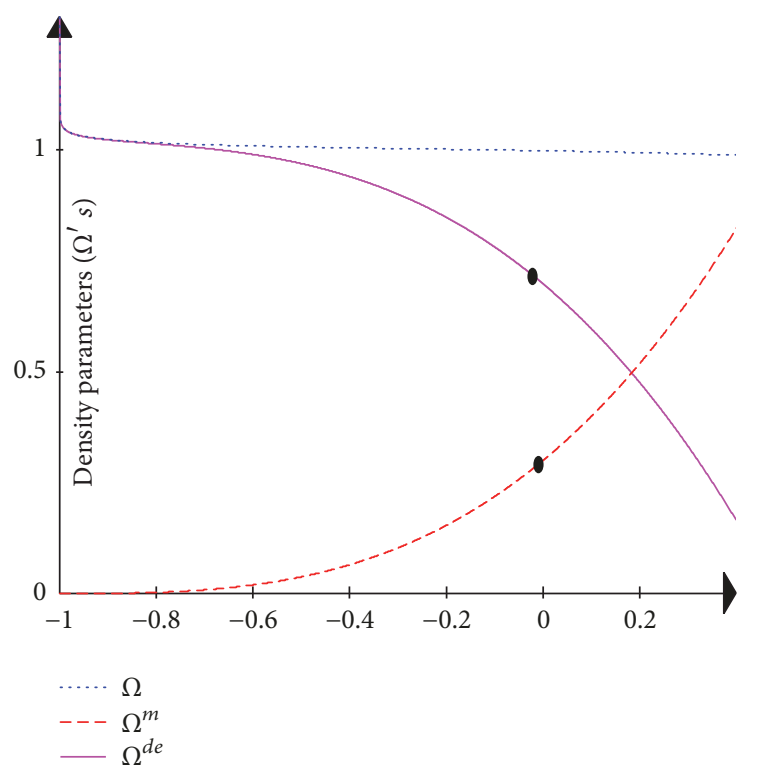

FIgURE 2: The plot of total energy densities versus redshift $z$ for $\alpha=$ $1, \beta=0.01, \Omega_{0}^{m}=0.3, \omega^{m}=0, \bar{\omega}=2$, and $K=0.01$.

these parameters. It is observed that, for sufficiently large time, the overall density parameter $(\Omega)$ approaches to 1 . Therefore the model predicts a flat universe at late time. It is interesting to note that the value of $\beta$ (i.e., BD theory) brings impact on the evolution of the densities (Figure 2). The dark energy density parameter is increasing whereas the matter density parameter is decreasing. It is also clear that the value of dark energy density parameter is greater than the matter density parameter. Thus, the universe is dominated by dark energy throughout the evolution.

3.2. Interacting Dark Energy and Dark Matter. In this section, we have considered interaction between DE and DM. For this purpose we can write the energy conservation equation (30) as

$$
\dot{\rho}^{m}+3 \frac{\dot{a}}{a}\left(1+\omega^{m}\right) \rho^{m}=Q
$$

and

$$
\dot{\rho}^{d e}+3 \frac{\dot{a}}{a}\left(1+\omega^{d e}\right) \rho^{d e}=-Q
$$

where $Q$ is interacting term.

To find the solution of coincidence problem, we have considered an energy transfer from dark energy to dark matter by assuming $Q>0$, which ensures that the second law of thermodynamics is fulfilled (Pavon and Wang [55]). The continuity equations (45) and (46) imply that the interaction term $Q$ should be proportional to inverse of time. Therefore, a first and natural candidate can be the Hubble parameter $H$ multiplied with the energy density. Following Amendola et al. [56] and Guo et al. [57], we consider

$$
Q=3 H \sigma \rho^{m}
$$

where $\sigma>0$ is coupling coefficient which can be considered as a constant or function of redshift $z$.

Using (45), we obtain the energy density of dark matter as

$$
\begin{aligned}
\rho^{m} & =\rho_{0}^{m} a^{-3\left(1+\omega^{m}-\sigma\right)} \\
& =\rho_{0}^{m}(1+z)^{3\left(1+\omega^{m}-\sigma\right)},
\end{aligned}
$$

where $\rho_{0}^{m}$ is an integrating constant.

Using (48) in (29), we obtain the energy density of DE as

$$
\begin{aligned}
\rho^{d e} & =D H^{2} \alpha a^{\beta}-\rho_{0}^{m} a^{-3\left(1+\omega^{m}-\sigma\right)}-K \alpha^{-1} a^{-(\beta+6)} \\
= & \left(1+(1+z)^{2}\right)\left(D \alpha(1+z)^{-\beta}-3 \Omega_{0}^{m}(1+z)^{3\left(1+\omega^{m}-\sigma\right)}\right) \\
& -K \alpha^{-1}(1+z)^{(\beta+6)} .
\end{aligned}
$$

Using (48) and (50) in (10), we obtain

$$
\omega^{d e}=\frac{H^{2}\left[(\beta+2) q-\left(\beta^{2}(1+\bar{\omega} / 2)+\beta+1\right)\right] \alpha a^{\beta}+K \alpha^{-1} a^{-(\beta+6)}-3 H^{2} \omega^{m} \Omega_{0}^{m} a^{-3\left(1+\omega^{m}-\sigma\right)}}{H^{2}\left(D \alpha a^{\beta}-3 \Omega_{0}^{m} a^{-3\left(1+\omega^{m}-\sigma\right)}\right)-K \alpha^{-1} a^{-(\beta+6)}} .
$$

This is the general form of the EoS parameter of DE in $\mathrm{BD}$ theory for interacting case. Here $q=-\ddot{a} / a H^{2}$ is the deceleration parameter.
Now using (16) in (52), we obtain the EoS parameter in terms of redshift as

$$
=-\frac{\left[(\beta+2)\left(1 /\left(1+(1+z)^{2}\right)\right)+\left(\beta^{2}(1+\bar{\omega} / 2)+\beta+1\right)\right] \alpha(1+z)^{-\beta}-K \alpha^{-1}\left((1+z)^{(\beta+6)} /\left(1+(1+z)^{2}\right)\right)+3 \omega^{m} \Omega_{0}^{m}(1+z)^{3\left(1+\omega^{m}-\sigma\right)}}{D \alpha(1+z)^{-\beta}-K \alpha^{-1}\left((1+z)^{(\beta+6)} /\left(1+(1+z)^{2}\right)\right)-3 \Omega_{0}^{m}(1+z)^{3\left(1+\omega^{m}-\sigma\right)}} .
$$




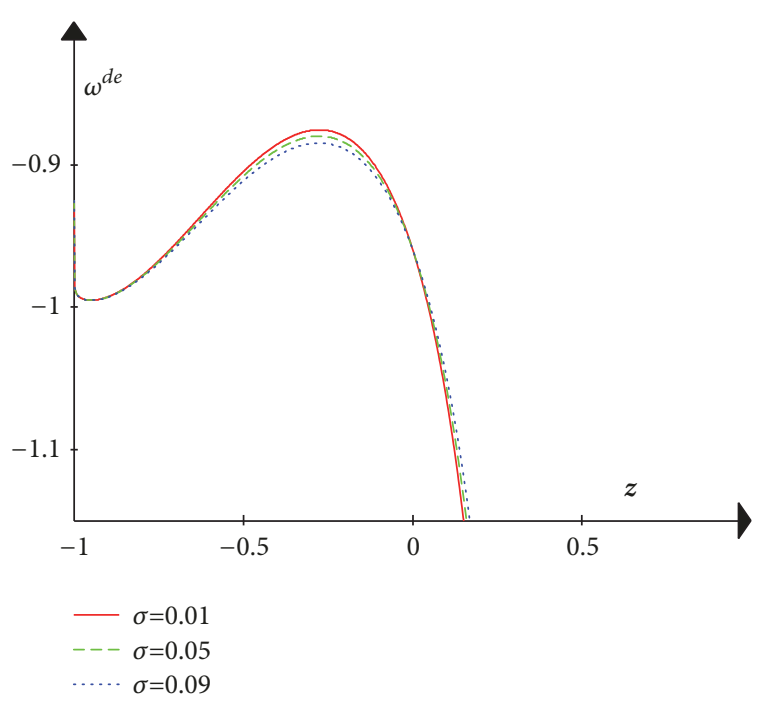

FIgURE 3: The plot of EoS parameter $\omega^{\text {de }}$ versus redshift $z$ for $\alpha=$ $1, \beta=0.01, \Omega_{0}^{m}=0.3, \omega^{m}=0, \bar{\omega}=2$, and vary $K=0.01,0.05$, 0.09 , resp.

The behavior of EoS parameter in terms of redshift $z$ is depicted in Figure 3. We fixed the parameters $\omega^{m}=0, K=$ 0.01 , and vary $\sigma$ as $0.01,0.05$, and 0.09 , respectively. This figure shows that, for all values of coupling constant $\sigma$, the EoS parameter of $\mathrm{DE}$ is varying in quintessence region, crosses the PDL, and varies in phantom region. At late time (i.e., at $z=-1$ ), the EoS parameter of DE $\omega^{d e} \approx-1$. Therefore we say that the cosmological constant is a suitable candidate to represent the behavior of DE in the derived model at late times.

The expression for matter energy density $\Omega^{m}$ and dark energy density $\Omega^{d e}$ are given by

$$
\begin{aligned}
\Omega^{m} & =\frac{\rho^{m}}{3 H^{2}} \\
& =\Omega_{0}^{m}(1+z)^{3\left(1+\omega^{m}-\sigma\right)},
\end{aligned}
$$

and

$$
\begin{aligned}
\Omega^{d e} & =\frac{\rho^{d e}}{3 H^{2}} \\
& =\frac{D \alpha}{3}(1+z)^{-\beta}-\frac{K \alpha^{-1}(1+z)^{(\beta+6)}}{3\left(1+(1+z)^{2}\right)} \\
& -\Omega_{0}^{m}(1+z)^{3\left(1+\omega^{m}-\sigma\right)} .
\end{aligned}
$$

Using (55) and (57), we obtain total energy density parameter

$$
\Omega=\Omega^{m}+\Omega^{d e}=\frac{D \alpha}{3}(1+z)^{-\beta}-\frac{K \alpha^{-1}(1+z)^{(\beta+6)}}{3\left(1+(1+z)^{2}\right)},
$$

and this equation is same as (44). The variation of density parameter $\Omega^{m}$ and $\Omega^{d e}$ with redshift $z$ is depicted in Figure 4 . In this figure the dot denotes the current value of these

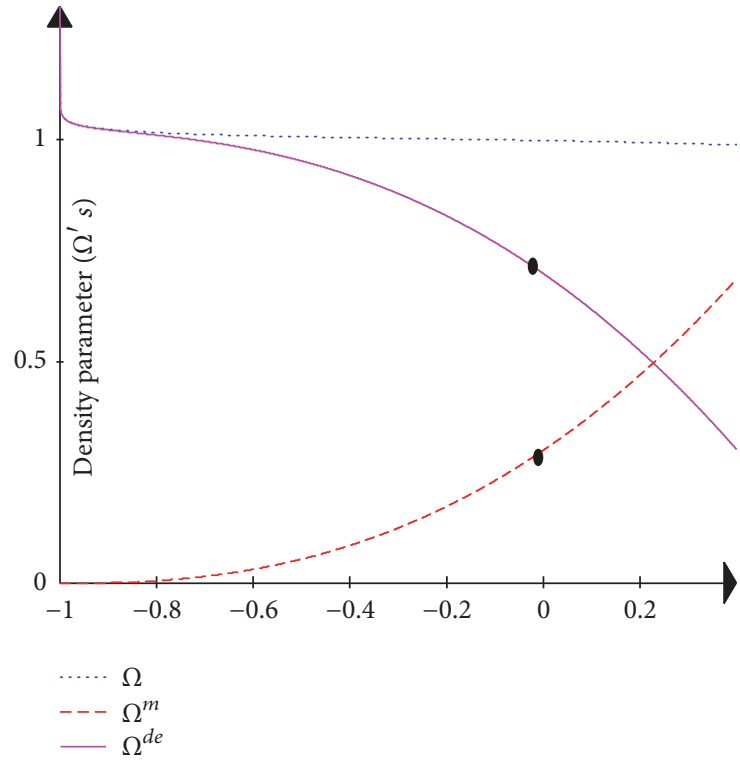

FIGURE 4: The plot of total energy densities versus redshift $z$ for $\alpha=$ $1, \beta=0.01, \Omega_{0}^{m}=0.3, \omega^{m}=0 \bar{\omega}=2, K=0.01$, and $\sigma=0.18$.

parameters. Hence we observed that in interacting case the density parameter has the same properties as in noninteracting case. From Figures 2 and 4, we observed that with interaction DE and DM follow one another. This means that in the recent history of the universe $\mathrm{DE}$ is being transformed into DM and the fluctuations do get more effective in the past. Note that the stronger the interaction is, the more effectively structures will have been formed in the past.

\section{Physical Acceptability and Stability Analysis}

To find the stability condition of corresponding models, we use squared speed of sound $\left(v_{s}^{2}\right)$. A positive value of squared speed of sound $\left(v_{s}^{2}\right)$ represents a stable model whereas the negative value of squared speed of sound $\left(v_{s}^{2}\right)$ indicates the instability of model. A squared speed of sound $\left(v_{s}^{2}\right)$ is defined as

$$
v_{s}^{2}=\frac{\dot{p}^{d e}}{\dot{\rho}^{d e}} .
$$

The squared speed of sound for non-interacting and interacting models are, respectively, given by

$$
\begin{aligned}
v_{s}^{2} & =\left\{\alpha \beta(z+1)^{-1-\beta}\left(1+(1+z)^{2}\right)\right. \\
& \cdot\left[\beta^{2}\left(\frac{\bar{\omega}}{2}+1\right)+\frac{\beta+2}{1+(1+z)^{2}}+\beta+1\right] \\
& -\alpha(2 z+2)(1+z)^{-\beta} \\
& \cdot\left[\beta^{2}\left(\frac{\bar{\omega}}{2}+1\right)+\frac{\beta+2}{1+(1+z)^{2}}+\beta+1\right]
\end{aligned}
$$




$$
\begin{aligned}
& +\frac{\alpha(\beta+2)(2 z+2)(1+z)^{-\beta}}{1+(1+z)^{2}}+K \alpha^{-1}(\beta+6) \\
& \cdot(1+z)^{(\beta+5)}-3 \omega^{m} \Omega_{0}^{m}(1+z)^{3 \omega^{m}+2} \\
& \left.\cdot\left[3 \omega^{m}\left(1+(1+z)^{2}\right)+5 z^{2}+10 z+8\right]\right\} / \\
& \left\{-\alpha D(z+1)^{-1-\beta}\left[2(1+z)^{2}-\beta\left(1+(1+z)^{2}\right)\right]\right. \\
& -K \alpha^{-1}(\beta+6)(1+z)^{(\beta+5)}-3 \Omega_{0}^{m}(1+z)^{3 \omega^{m}+2} \\
& \left.\cdot\left[3 \omega^{m}\left(1+(1+z)^{2}\right)+5 z^{2}+10 z+8\right]\right\}
\end{aligned}
$$

and

$$
\begin{aligned}
v_{s}^{2} & =\left\{\alpha \beta ( z + 1 ) ^ { - 1 - \beta } ( 1 + ( 1 + z ) ^ { 2 } ) \left[\beta^{2}\left(\frac{\bar{\omega}}{2}+1\right)\right.\right. \\
& \left.+\frac{\beta+2}{1+(1+z)^{2}}+\beta+1\right]-\alpha(2 z+2)(1+z)^{-\beta} \\
& \cdot\left[\beta^{2}\left(\frac{\bar{\omega}}{2}+1\right)+\frac{\beta+2}{1+(1+z)^{2}}+\beta+1\right] \\
& +\frac{\alpha(\beta+2)(2 z+2)(1+z)^{-\beta}}{1+(1+z)^{2}}+K \alpha^{-1}(\beta+6)(1 \\
& +z)^{(\beta+5)}-3 \omega^{m} \Omega_{0}^{m}\left[\left(3 \omega^{m}-3 \sigma+3\right)\left(1+(1+z)^{2}\right)\right. \\
& \left.\left.\cdot(1+z)^{3 \omega^{m}-3 \sigma+2}+(2 z+2)(z+1)^{3 \omega^{m}-3 \sigma+3}\right]\right\} / \\
& \left\{-\alpha D(z+1)^{-1-\beta}\left[2(1+z)^{2}-\beta\left(1+(1+z)^{2}\right)\right]\right. \\
& -K \alpha^{-1}(\beta+6)(1+z)^{(\beta+5)} \\
& -3 \Omega_{0}^{m}\left[\left(3 \omega^{m}-3 \sigma+3\right)\left(1+(1+z)^{2}\right)\right. \\
& \left.\left.\cdot(1+z)^{3 \omega^{m}-3 \sigma+2}+(2 z+2)(z+1)^{3 \omega^{m}-3 \sigma+3}\right]\right\}
\end{aligned}
$$

From Figures 5 and 6, it is observed that in our noninteracting and interacting models the sound speed remains positive (i.e., $v_{s}^{2}>0$ ); hence our models shows the stability throughout the evolution of the universe.

Secondly, the plot of weak energy condition (WEC), dominant energy condition (DEC), and strong energy condition (SEC) for non-interacting and interacting cases is shown in Figures 7 and 8, respectively. It is observed that, in noninteracting and interacting cases, the energy conditions obey the following restrictions:

$$
\begin{aligned}
& \text { (i) } \rho^{d e} \geq 0 \\
& \text { (ii) } \rho^{d e}+p^{d e} \geq 0, \\
& \text { (iii) } \rho^{d e}+3 p^{d e} \leq 0
\end{aligned}
$$

From Figures 7 and 8 and above expressions, we observed that the WEC and DEC are satisfied for non-interacting and interacting cases whereas the SEC is violated in entire

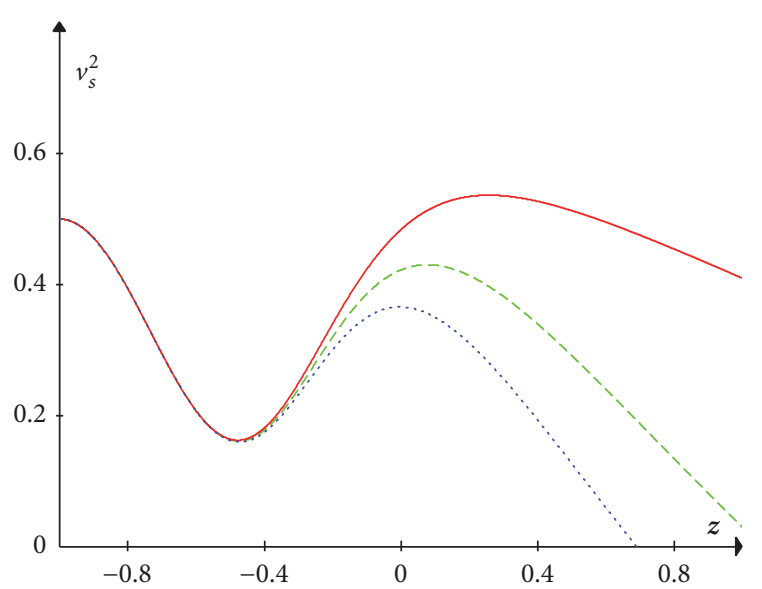

$$
\begin{aligned}
&-K=0.01 \\
&---K=0.05 \\
& \cdots \cdots K=0.09
\end{aligned}
$$

FIgURE 5: The plot of sound speed $v_{s}^{2}$ versus redshift $z$ in noninteracting case for $\alpha=1, \beta=1, \Omega_{0}^{m}=0.3, \bar{\omega}=1 \omega^{m}=0$, and vary $K=0.01,0.05,0.09$, resp.

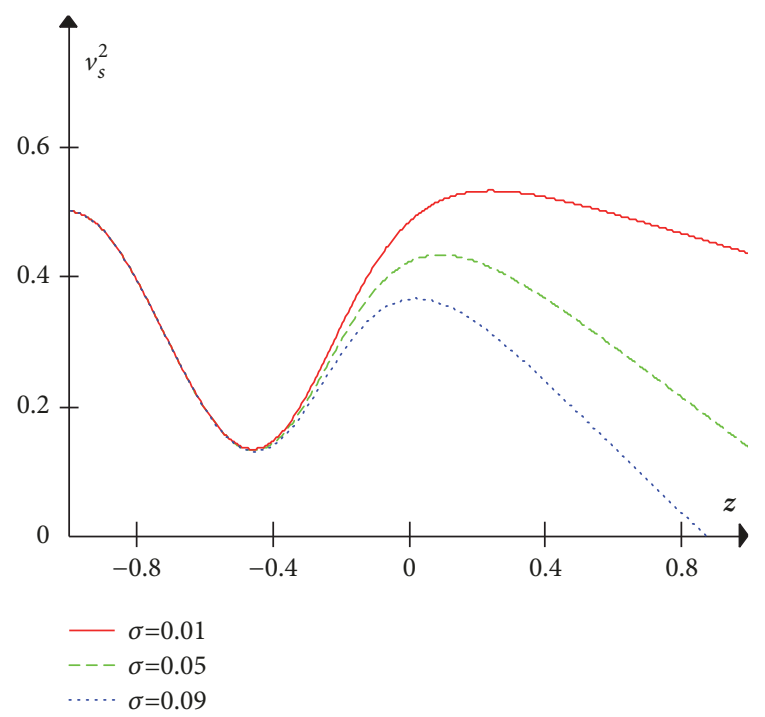

FIGURE 6: The plot of sound speed $v_{s}^{2}$ versus redshift $z$ in interacting case for $\alpha=1, \beta=1, \Omega_{0}^{m}=0.3, \bar{\omega}=1, \omega^{m}=0, K=0.01$, and vary $\sigma=0.01,0.05,0.09$, resp.

evolution of the universe in non-interacting and interacting scenario.

Therefore, on the basis of above discussion and analysis, our corresponding models are physically acceptable.

\section{Anisotropy Parameter $(\Delta)$}

The anisotropy parameter of expansion $(\Delta)$ is defined as

$$
\Delta=\frac{1}{3 H^{2}} \sum_{i=1}^{3}\left(H_{i}-H\right)^{2}
$$




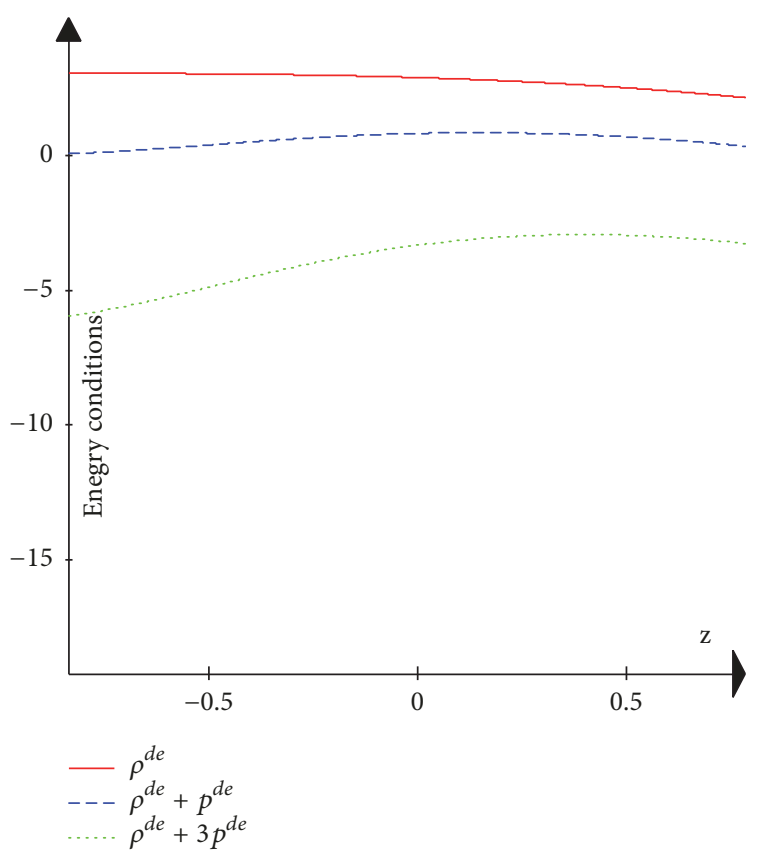

FIGURE 7: The plot of the weak $\rho^{d e} \geq 0$, dominant $\rho^{d e}+p^{d e} \geq 0$, and strong $\rho^{d e}+3 p^{d e} \geq 0$ energy conditions versus redshift $z$ for non-interacting scenario.

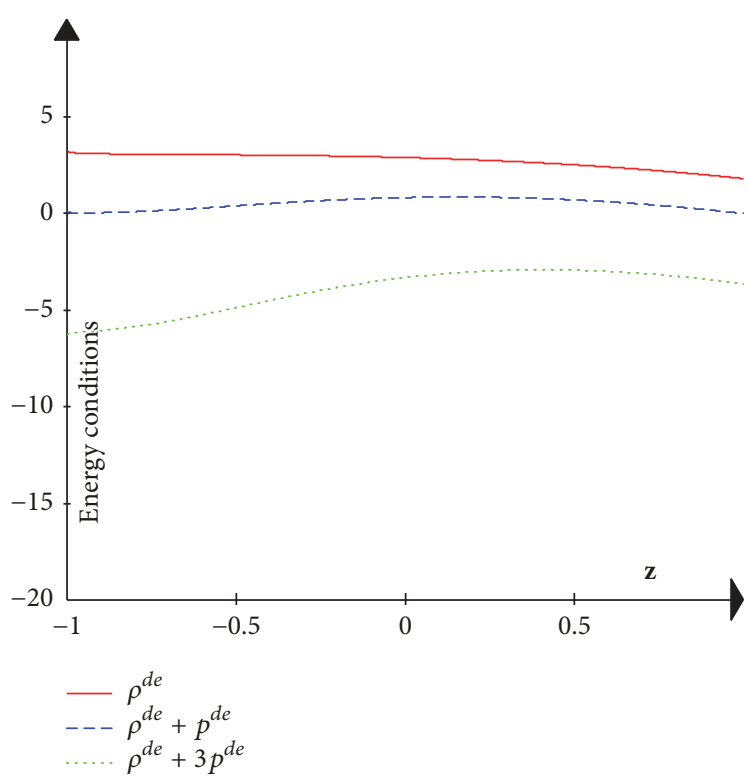

FIGURE 8: The plot of the weak $\rho^{d e} \geq 0$, dominant $\rho^{d e}+p^{d e} \geq 0$, and strong $\rho^{d e}+3 p^{d e} \geq 0$ energy conditions versus redshift $z$ for interacting scenario.

$$
=\frac{\left(b_{1}^{2}+b_{2}^{2}+b_{3}^{2}\right)}{3 H^{2} \phi^{2} a^{6}}=\frac{\left(b_{1}^{2}+b_{2}^{2}+b_{3}^{2}\right)(1+z)^{2(\beta+3)}}{3 \alpha^{2}\left(1+(1+z)^{2}\right)} \text {. }
$$

The anisotropy parameter versus redshift $z$ is depicted in Figure 9. It is observed that the anisotropy parameter is decreasing function and dies out at late time $(z=-1)$. Hence

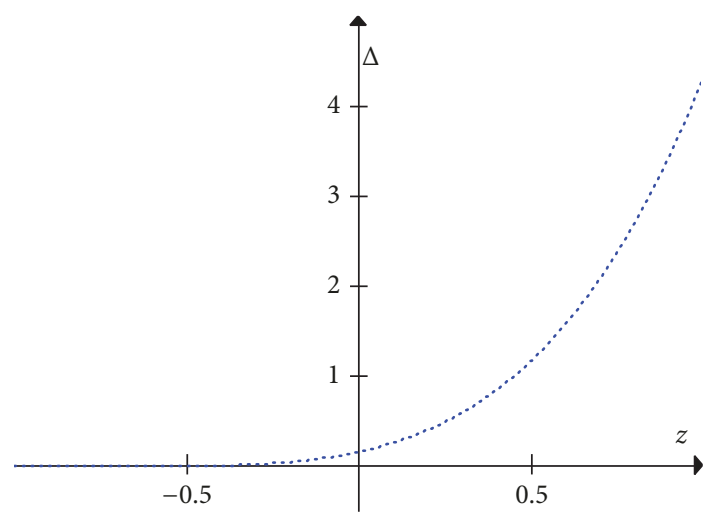

FIGURE 9: The plot of anisotropy parameter $(\Delta)$ versus redshift $z$.

the model reaches isotropy which matches with the recent observations as the universe is isotropic at large scale.

\section{Statefinder Parameters}

In order to get an accurate analysis to discriminate among the dark energy models Sahni et al. [58] proposed a new geometrical diagnostic named as statefinder pair $\{r, s\}$ which is constructed from scale factor $(a)$ and its derivative up to third order. The statefinder parameters are defined as

$$
\begin{aligned}
& r=\frac{\dddot{a}}{a H^{3}}, \\
& s=\frac{r-1}{3(q-1 / 2)} .
\end{aligned}
$$

These parameters allow us to characterize the properties of $D E$. Using these parameters one can describe the well-known region as follows: $(r, s)=(1,0)$ indicates $\Lambda C D M$ limit and $(r, s)=(1,1)$ indicates CDM limit, while $s>0$ and $r<$ 1 correspond to region of phantom and quintessence dark energy era. The relation between statefinder parameters for our models is obtain as

$$
\begin{aligned}
& r=\frac{1}{1+(1+z)^{2}}, \\
& s=\frac{2(1+z)^{2}}{3\left(1+(1+z)^{2}\right)} .
\end{aligned}
$$

From (64) and (65) a relation between parameters $r$ and $s$ is given by

$$
s=\frac{2(1-r)}{3} .
$$

The variation of parameter $s$ versus $r$ is plotted in Figure 10.

From Figure 9, it is seen that the curve passes through the point $(r=1, s=0)$; thus it can be concluded that our model corresponds to $\Lambda C D M$ model. 


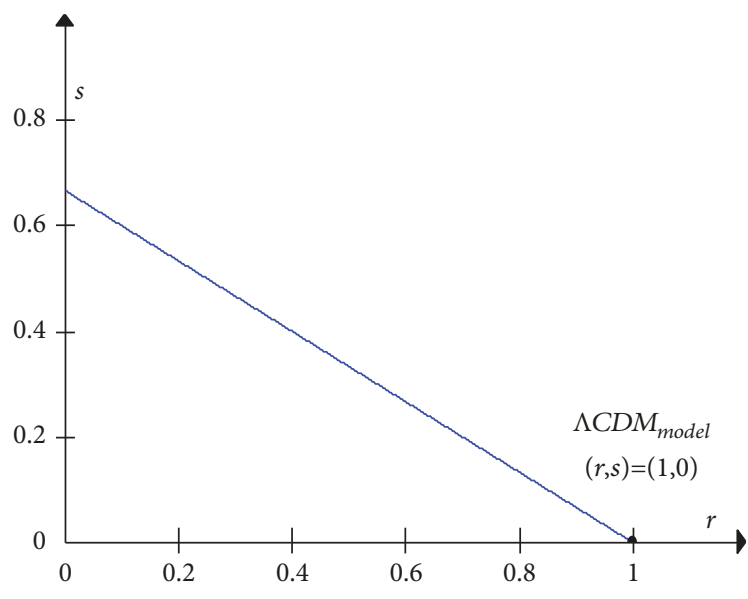

FIGURE 10: The plot of statefinder parameters $s$ versus $r$.

\section{Conclusion}

In this paper, we have studied interacting and non-interacting $\mathrm{DE}$ and DM in the anisotropic Bianchi type-I universe in the framework of Brans-Dicke theory of gravitation. To obtain the exact solutions of Brans-Dicke field equations we have used (i) the power-law relation between ' $\phi$ 'and ' $a$ ' and (ii) the average scale factor in terms of redshift. In noninteracting and interacting cases the general form of EoS parameter is derived. Then using the scale factor in terms of redshift, results are examined. We have discussed the physical acceptability and stability of our models. It is found that our models are physically acceptable and stable.

In non-interacting case, the EoS parameter of $\mathrm{DE}$ is varying in quintessence region and crossing PDL depending on the values of constant $K$ whereas in interacting case for all small values of coupling constant $\sigma$ the EoS parameter of DE varies in quintessence region; it crosses the PDL and varies in phantom region. However, at late time (i.e., at $z=-1$ ) the EoS parameter of both the cases tends to a cosmological constant $\left(\omega^{d e}=-1\right)$. The anisotropy parameter of expansion $(\Delta)$ is calculated and studied. It is observed that for $z=1$ (i.e., at initial time) it is infinite but at $z=0$ (i.e., at present time) it is closer to zero and at $z=-1$ it completely dies out which matches with the present day observations, which indicate that our model at present time $(z=0)$ is closer to the de Sitter model and attains complete de Setter model at $z=-1$; i.e., we can say that the Bianchi type-I space-time reduces to flat FRW (isotropic) soon after the inflation. As mentioned in Carroll et al. [59], this ensures that there is no Big rip singularity; rather, the universe eventually settles into a de Sitter phase. Finally the statefinder diagnostic pair $\{r, s\}$ is adapted to differentiate the different forms of DE. The trajectories in the $\{r, s\}$ plane corresponds to the $\Lambda C D M$ model (as shown in Figure 10).

\section{Data Availability}

No data were used to support this study.

\section{Conflicts of Interest}

The authors declare that they have no conflicts of interest.

\section{References}

[1] A. G. Riess et al., "Observational evidence from supernovae for an accelerating universe and a cosmological constant," The Astrophysical Journal, vol. 116, p. 1009, 1998.

[2] S. Perlmutter, G. Aldering1, and G. Goldhaber, "Measurements of $\Omega$ and $\Lambda$ from 42 high-redshift supernovae," The Astrophysical Journal, vol. 517, p. 565, 1999.

[3] C. L. Bennett et al., "First year Wilkinson Microwave Anisotropy Probe (WMAP) observations: preliminary maps and basic results," The Astrophysical Journal Supplement Series, vol. 148, p. 1, 2003.

[4] D. N. Spergel et al., "First year Wilkinson Microwave Anisotropy Probe (WMAP) observations: determination of cosmological parameters," The Astrophysical Journal Supplement Series, vol. 148, p. 175, 2003.

[5] M. Tegmark, M. R. Blanton, and M. A. Strauss, "The threedimensional power spectrum of galaxies from the Sloan digital sky survey," The Astrophysical Journal, vol. 606, no. 2, pp. 702$740,2004$.

[6] M. Tegmark et al., "Cosmological parameters from SDSS and WMAP," Physical Review D, vol. 69, Article ID 103501, 2004.

[7] U. Seljak et al., "Cosmological parameter analysis including SDSS Ly $\alpha$ forest and galaxy bias: constraints on the primordial spectrum of fluctuations, neutrino mass, and dark energy," Physical Review D, vol. 71, Article ID 103515, 2005.

[8] J. K. Adelman-McCarthy et al., "The fourth data release of the sloan digital sky survey," The Astrophysical Journal Supplement Series, vol. 162, p. 38, 2006.

[9] R. A. Knop et al., "New constraints on $\Omega M, \Omega \Lambda$, and w from an independent set of eleven high-redshift supernovae observed with HST," The Astrophysical Journal, vol. 598, p. 102, 2003.

[10] S. W. Allen et al., "Constraints on dark energy from Chandra observations of the largest relaxed galaxy clusters," Monthly Notices of the Royal Astronomical Society, vol. 353, p. 457, 2004.

[11] S. Ram et al., "Kantowski- Sachs universe with anisotropic dark energy in Lyra geometry," Chinese Journal of Physics, p. 1, 2016.

[12] S. Ram et al., Chinese Journal of Physics, vol. 54, p. 953, 2016.

[13] S. D. Katore and D. V. Kapse, "Dynamics of Bianchi type-VI0 holographic dark energy models in general relativity and Lyra's geometry," Pramana-Journal of Physics, vol. 88, p. 30, 2017.

[14] D. R. K Reddy, "Anisotropic holographic dark energy model in Bianchi type-VI0 universe in a scalar-tensor theory of gravitation," Astrophysics and Space Science, vol. 361, p. 349, 2016.

[15] C. R. Mahanta and N. Sharma, "Anisotropic ghost dark energy cosmological model with hybrid expansion law," New Astronomy, vol. 57, pp. 70-75, 2017.

[16] L. P. Chimento, "Dark energy, dissipation and the coincidence problem," Physical Review D, vol. 67, Article ID 087302, 2003.

[17] N. Dalal, K. Abazajian, E. Jenkins, and A. V. Manohar, "Testing the cosmic coincidence problem and the nature of dark energy," Physical Review Letters, vol. 87, no. 14, Article ID 141302, 2001.

[18] M. Jamil and M. A. Rashid, "Interacting modified variable Chaplygin gas in a non-flat universe," The European Physical Journal C, vol. 58, p. 111, 2008.

[19] M. Jamil and M. A. Rashid, "Constraining the coupling constant between dark energy and dark matter," The European Physical Journal C, vol. 60, p. 141, 2009.

[20] O. Bertolami, F. Gil Pedro, and M. Le Delliou, "Dark energydark matter interaction and putative violation of the equivalence principle from the Abell cluster A586," Physics Letters B, vol. 654, no. 5-6, pp. 165-169, 2007. 
[21] M. Le Delliou, O. Bertolami, and F. Gil Pedro, "Dark energydark matter interaction from the Abell cluster A586 and violation of the equivalence principle," AIP Conference Proceedings, vol. 957, p. 421, 2007.

[22] M. S. Berger and H. Shojaei, "Interacting dark energy model for the expansion history of the Universe," Physical Review D: Particles, Fields, Gravitation and Cosmology, vol. 74, no. 4, Article ID 043530, 2006.

[23] X. Zhang, "An interacting two-fluid scenario for quintom dark energy," Communications in Theoretical Physics, vol. 4, p. 762, 2005.

[24] X. Zhang, "Statefinder diagnostic for coupled quintessence," Physics Letters B, vol. 611, p. 1, 2005.

[25] W. Zimdahl and D. Pavón, "Statefinder parameters for interacting dark energy," General Relativity and Gravitation, vol. 36, no. 6, pp. 1483-1491, 2004.

[26] A. Pradhan, H. Amirhashchi, and B. Saha, "An interacting and non-interacting two-fluid scenario for dark energy in FRW universe with constant deceleration parameter," Astrophysics and Space Science, vol. 333, p. 343, 2011.

[27] A. Pradhan, H. Amirhashchi, and B. Saha, "Bianchi typeI anisotropic dark energy model with constant deceleration parameter," International Journal of Theoretical Physics, vol. 50, p. 2923, 2011.

[28] B. Saha, H. Amirhashchi, and A. Pradhan, "Two-fluid scenario for dark energy models in an FRW universe-revisited," Astrophysics and Space Science, vol. 342, p. 257, 2012.

[29] H. Amirhashchi, "Two-fluid dark energy models in Bianchi type-III universe with variable deceleration parameter," International Journal of Theoretical Physics, vol. 52, p. 2735, 2013.

[30] H. Amirhashchi et al., "Interacting two-fluid viscous dark energy models in a non-flat universe," Research in Astronomy and Astrophysics, vol. 13, p. 2, 2013.

[31] H. Amirhashchi, "Bianchi type-I model, dark energy, statefinder," Astrophysics and Space Science, vol. 351, p. 641, 2014.

[32] H. Amirhashchi et al., "Interacting and non-interacting twofluid atmosphere for dark energy in FRW universe," Electronic Journal of Theoretical Physics, vol. 11, p. 109, 2014.

[33] H. Amirhashchi, S. N. A. Qazi, and H. Zainuddin, "An interacting scenario for dark energy in a Bianchi type-I universe," Research in Astronomy and Astrophysics, vol. 14, no. 11, pp. 13831392, 2014.

[34] K. S. Adhav et al., "Interacting dark matter and holographic dark energy in an anisotropic universe," Astrophysics and Space Science, vol. 353, p. 249, 2014.

[35] K. S. Adhav et al., "Interacting dark matter and holographic dark energy in Bianchi type-V universe," Astrophysics and Space Science, vol. 359, p. 24, 2015.

[36] V. Fayaz, "Anisotropic universe and thermodynamical illustration of the interacting generalized ghost dark energy," Canadian Journal of Physics, vol. 94, p. 540, 2016.

[37] C. Brans and R. H. Dicke, "Mach's principle and a relativistic theory of gravitation," Physical Review A: Atomic, Molecular and Optical Physics, vol. 124, no. 3, pp. 925-935, 1961.

[38] V. U. M. Rao and U. Y. Divya Prasanthi, "Bianchi type VI0 generalized ghost pilgrim dark energy model in Brans-Dicke theory of gravitation," Canadian Journal of Physics, vol. 94, p. 1040, 2016.

[39] S. Sarkar, "Holographic dark energy model with linearly varying deceleration parameter and generalised Chaplygin gas dark energy model in Bianchi type-I universe," Astrophysics and Space Science, vol. 34, p. 985, 2014.

[40] S. Sarkar, "Holographic dark energy with linearly varying deceleration parameter and escaping big rip singularity of the Bianchi type-V universe," Astrophysics and Space Science, vol. 352, p. 859, 2014.

[41] S. D. Katore et al., "Hypersurface homogeneous space time with anisotropic dark energy in Brans Dicke theory of gravitation," Communications in Theoretical Physics, vol. 62, p. 5, 2014.

[42] K. P. Singh and M. Dewri, "Robertson-Walker model universe interacting with electromagnetic field and Brans-Dicke field in presence of hybrid scale factor," Chinese Journal of Physics, p. 1, 2016.

[43] D. R. K. Reddy et al., "Kaluza-Klein dark energy model in BransDicke theory of gravitation," Astrophysics and Space Science, vol. 357, p. 31, 2015.

[44] D. R. K. Reddy et al., "Five dimensional spherically symmetric minimally interacting holographic dark energy model in Brans-Dicke theory," Astrophysics and Space Science, vol. 361, p. 123, 2016.

[45] H. Amirhashchi, "Probing dark energy in the scope of a Bianchi type I spacetime," Physical Review D, vol. 97, Article ID 063515, 2018.

[46] H. Amirhashchi and S. Amirhashchi, "Comparison Between Anisotropic and Isotropic Dark Energy Models Through Recent Observations," https://arxiv.org/abs/1802.04251.

[47] H. Amirhashchi and S. Amirhashchi, "Current Constraints on Anisotropic and Isotropic Dark Energy Models Using Gaussian Processes," https://arxiv.org/abs/1803.08447.

[48] C. Patrignani et al., "Review of Particle Physics," Chinese Physics C, vol. 40, no. 2016, 2017, (2017 update), 100001.

[49] V. U. M. Rao et al., "FRW holographic dark energy cosmological model in BransDicke theory of gravitation," Prespacetime Journal, vol. 6, p. 961, 2015.

[50] T. Harko and F. S. N. Lobo, "Irreversible thermodynamic description of interacting dark energy-dark matter cosmological models," Physical Review D: Particles, Fields, Gravitation and Cosmology, vol. 87, Article ID 044018, 2013.

[51] L. O. Pimentel, "Exact cosmological solutions in the scalartensor theory with cosmological constant," Astrophysics and Space Science, vol. 112, no. 1, pp. 175-183, 1985.

[52] V. B. Johri and K. Desikan, "Cosmological models with constant deceleration parameter in Brans-Dicke theory," General Relativity and Gravitation, vol. 26, no. 12, pp. 1217-1232, 1994.

[53] H. Amirhashchi, "Phantom instability of viscous dark energy in anisotropic space-time," Astrophysics and Space Science, vol. 345, p. 439, 2014.

[54] C.-M. Chen and W. F. Kao, "Stability analysis of anisotropic inflationary cosmology," Physical Review D: Particles, Fields, Gravitation and Cosmology, vol. 64, no. 12, Article ID 124019, 6 pages, 2001.

[55] D. Pavon and B. Wang, "Le Châtelier-Braun principle in cosmological physics," General Relativity and Gravitation, vol. 41, no. 1, pp. 1-5, 2009.

[56] L. Amendola, G. C. Campos, and R. Rosenfeld, "Consequences of dark matter-dark energy interaction on cosmological parameters derived from type Ia supernova data," Physical Review D: Particles, Fields, Gravitation and Cosmology, vol. 75, no. 8, Article ID 083506, 2007.

[57] Z. Guo, N. Ohta, and S. Tsujikawa, "Probing the coupling between dark components of the universe," Physical Review D: 
Particles, Fields, Gravitation and Cosmology, vol. 76, no. 2, Article ID 023508, 2007.

[58] V. Sahni, T. D. Saini, A. A. Starobinsky, and U. Alam, "Statefinder-a new geometrical diagnostic of dark energy," Journal of Experimental and Theoretical Physics Letters, vol. 77, no. 5, pp. 201-206, 2003.

[59] S. M. Carroll, M. Hoffman, and M. Trodden, "Can the dark energy equation-of-state parameter $w$ be less than -1?" Physical Review D: Particles, Fields, Gravitation and Cosmology, vol. 68, Article ID 023509, 2003. 

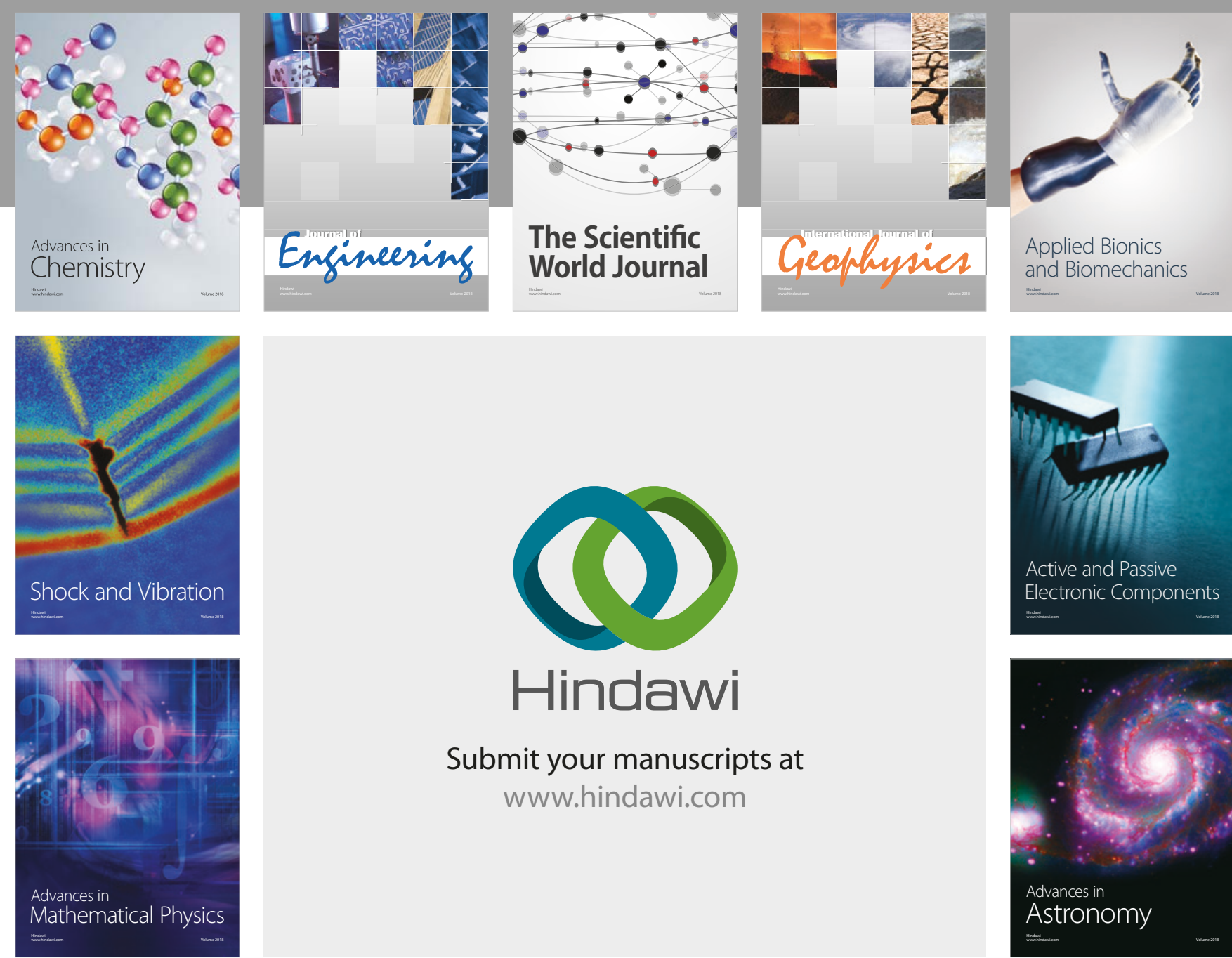

Submit your manuscripts at

www.hindawi.com

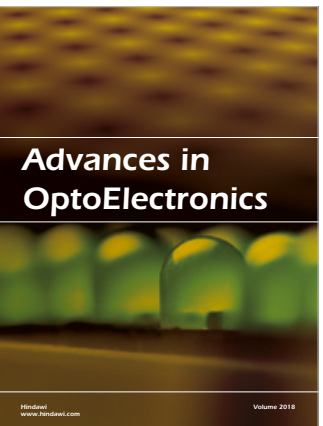

\section{Rotcting Machinery}
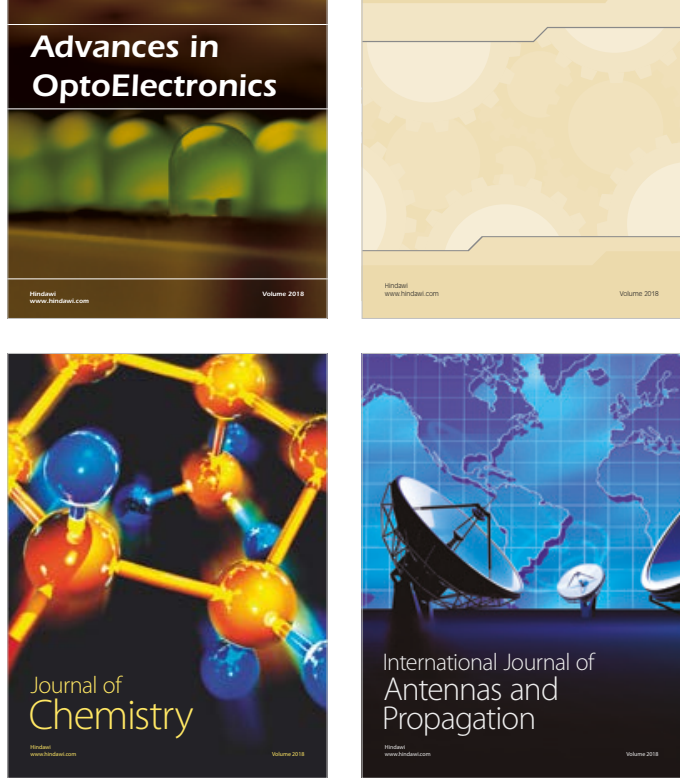

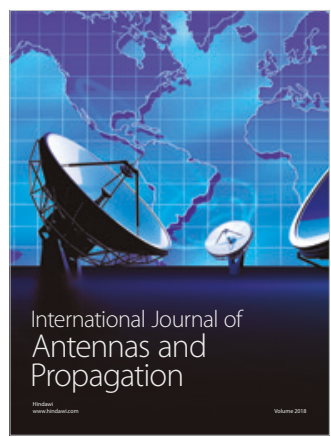

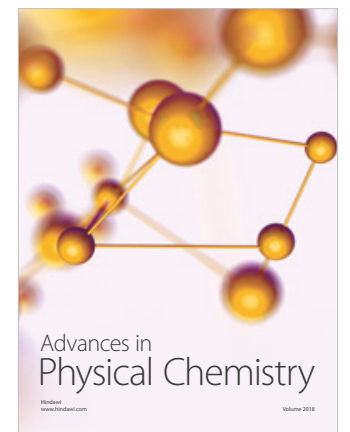

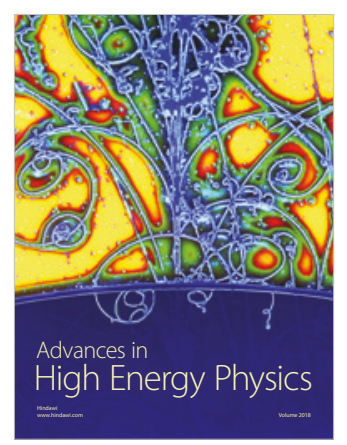

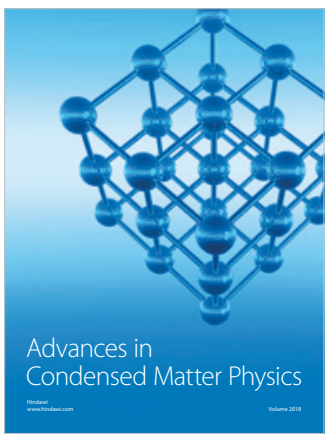

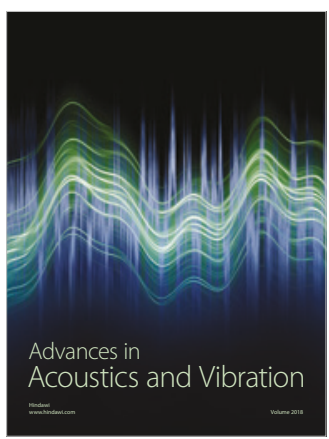

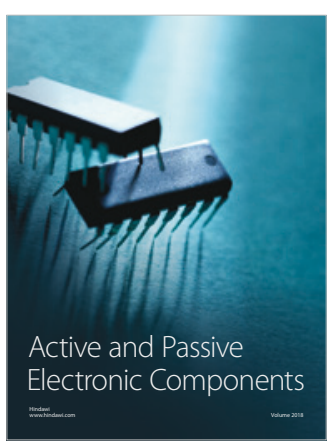
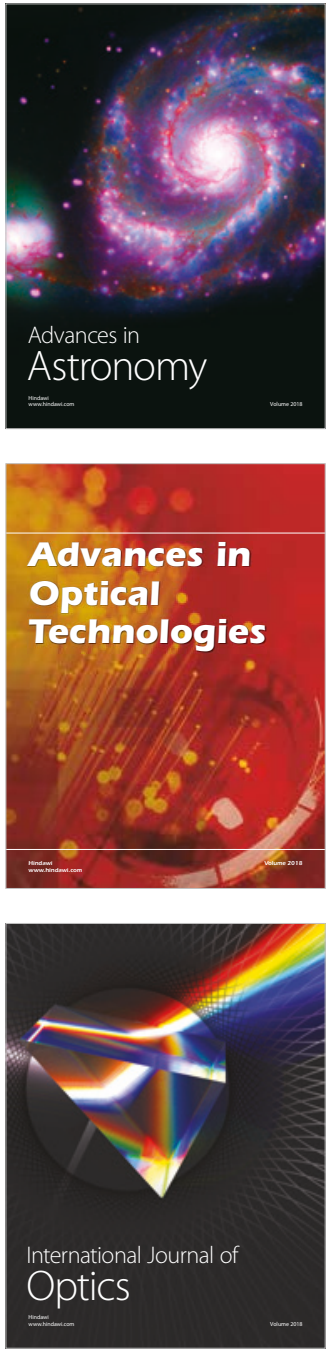